EPJ Web of Conferences 73, 02003 (2014)

DOI: $10.1051 /$ epjconf/20147302003

(C) Owned by the authors, published by EDP Sciences, 2014

\title{
Charged hadron lepto-production off unpolarized protons and deuterons at HERMES
}

\author{
Gevorg Karyan ${ }^{\mathrm{a}}$ \\ Alikhanyan National Science Laboratory, Yerevan, Armenia
}

\begin{abstract}
The HERMES collaboration has measured charge-separated pion and kaon multiplicities in semi-inclusive deep-inelastic scattering using a $27.6 \mathrm{GeV}$ electron or positron beam scattering off a hydrogen or deuterium target. The results are presented as functions of the Bjorken variable $x_{B}$, the negative squared four-momentum transfer $Q^{2}$, a hadron fractional energy $z$ and the hadron's transverse momentum. These data will be very useful to understand the quark-fragmentation process in deep-inelastic hadron electroproduction and will serve as crucial input in the understanding of charge and flavour separated fragmentation functions. Furthermore, it provides important information on the transverse-momentum dependence of hadron production.
\end{abstract}

\section{Introduction}

The measurement of the hadron multiplicities is one of the key ingredients to study the hadronization process. Due to a lack of a complete theory to describe a hadron production process, the input is needed for a phenomenological understanding of a quark fragmentation into hadrons. According to the factorization theorem [1] the cross section of hadron production in semi-inclusive deep inelastic leptonnucleon scattering (SIDIS) is described by parton distribution functions and fragmentation functions. The fragmentation functions should be universal [2]. They can be considered as global input information and can be compared with fragmentation functions extracted from different kind of precesses. The study of the hadronization process is an essential element of a complete picture of the interaction of quarks in Quantum ChromoDynamics (QCD), and is basic for understanding of the dynamics of quark-quark, gluon-gluon, and quark-gluon interactions.

\section{Data analysis}

The data are collected with the HERMES spectrometer using $27.6 \mathrm{GeV}$ electron or positron beam stored in HERA at DESY[3]. To select deep-inelastic scattered (DIS) lepton the following requirements are used for it's kinematics: $Q^{2}>1 \mathrm{GeV}^{2}, W^{2}>10 \mathrm{GeV}^{2}$ and $0.1<y=v / E<0.85$, where $W$ is the invariant mass of virtual photon-nucleon system, $v$ is a virtual photon energy and $E$ is the beam energy in a fixed target frame. The cut on the invariant mass suppresses the resonance contribution in the data,

\footnotetext{
${ }^{a}$ e-mail: gevkar@mail.desy.de (on behalf of the HERMES collaboration)
}

This is an Open Access article distributed under the terms of the Creative Commons Attribution License 4.0, which permits unrestricted use, distribution, and reproduction in any medium, provided the original work is properly cited. 


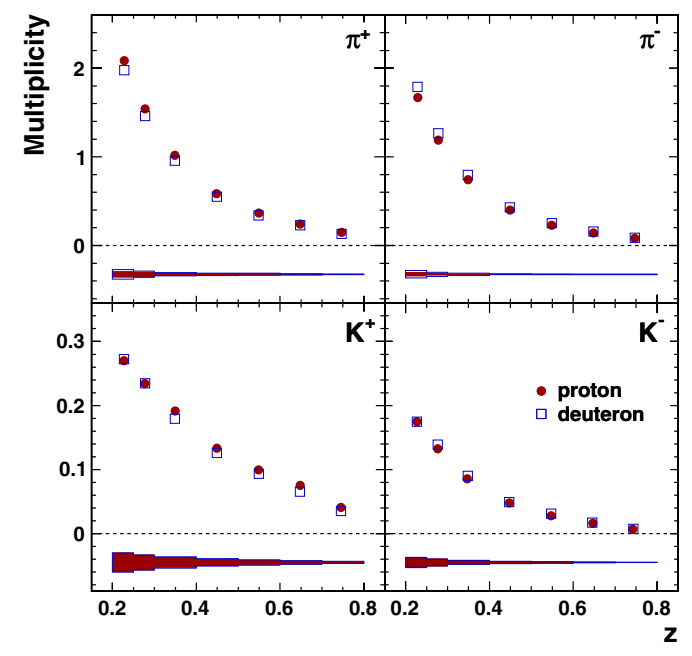

Figure 1. Multiplicities corrected for exclusive vector mesons as a function of $z$ from a proton target (full circles) and a deuteron target (empty squares). The error bars for the statistical uncertainties are too small to be visible. The systematic uncertainties are given by the error bands.

while the constrain on $y$ limits the magnitude of radiative corrections. For charged hadron identification, a dual-radiator ring-imaging Čerenkov detector (RICH) is used [4], which allows to identify pions and kaons. They are selected within the following kinematical conditions: $2<p_{h}<15 \mathrm{GeV}$, where $p_{h}$ is a hadron momentum. A $0.2<z<0.8$ cut is applied to exclude the region at high $z$, which is dominated by exclusive processes, and to suppress the target fragmentation region at low $z$. The experimental observable is the hadron multiplicity which is the hadron yield $N^{h}$ normalized to the DIS yield $N_{D I S}$ :

$$
M^{h}\left(x_{B}, Q^{2}, z, P_{h \perp}\right)=\frac{1}{\frac{d^{2} N_{D I S}\left(x_{B}, Q^{2}\right)}{d x_{B} d Q^{2}}} \cdot \int_{0}^{2 \pi} \frac{d^{5} N^{h}\left(x_{B}, Q^{2}, z, P_{h \perp}, \phi_{h}\right)}{d x_{B} d Q^{2} d z d P_{h \perp} d \phi_{h}} d \phi_{h} .
$$

The "Born multiplicities", i.e. the multiplicities at Born level are corrected for geometric acceptance, radiative effects and detector resolution. They are extracted from measured multiplicities binned in 3 dimensions: $\left(x_{B}, z, P_{h \perp}\right)$ or $\left(Q^{2}, z, P_{h \perp}\right)$. A simultaneous data binning in $x_{B}$ and $Q^{2}$ is not possible due to the strong correlation between the these two variables in the HERMES kinematical acceptance, A $\phi_{h}$ binning has been omitted because of limited statistical precision. However, a possible impact of the $\phi_{h}$ dependence of the unpolarized semi-inclusive cross section and its acceptance have been accounted for in the systematic uncertainties.

\section{Results}

The Born multiplicities, after correction for the exclusive vector-meson production, are presented in Figs. 1 and 2. In Fig. 1 they are shown for both proton (full circle) and deuteron targets (empty squares) as a function of the energy fraction $z$ in four panels corresponding to different types of final-state hadrons: $\pi^{+}, \pi^{-}, K^{+}, K^{-}$. The multiplicity ratio of $\pi^{+}$to $\pi^{-}$for the proton (deuteron) ranges from $1.2(1.1)$ in the first $z$ bin to 2.6 (1.8) in the last $z$ bin. These results can be attributed to the dominance of scattering off the $u$ quarks, reflecting the fact that the fragmentation process $u \rightarrow \pi^{+}$is favored, while the process $u \rightarrow \pi^{-}$is unfavored. With rising $z$, this effect is enhanced. For kaons, the $K^{+}$multiplicity for the proton is slightly larger than for the deuteron, while within errors the $K^{-}$multiplicities are equal. The ratio $\mathrm{K}^{+} / \mathrm{K}^{-}$rises from 1.5 to 5.7 (proton target) and from 1.3 to 4.6 (deuteron target) for 

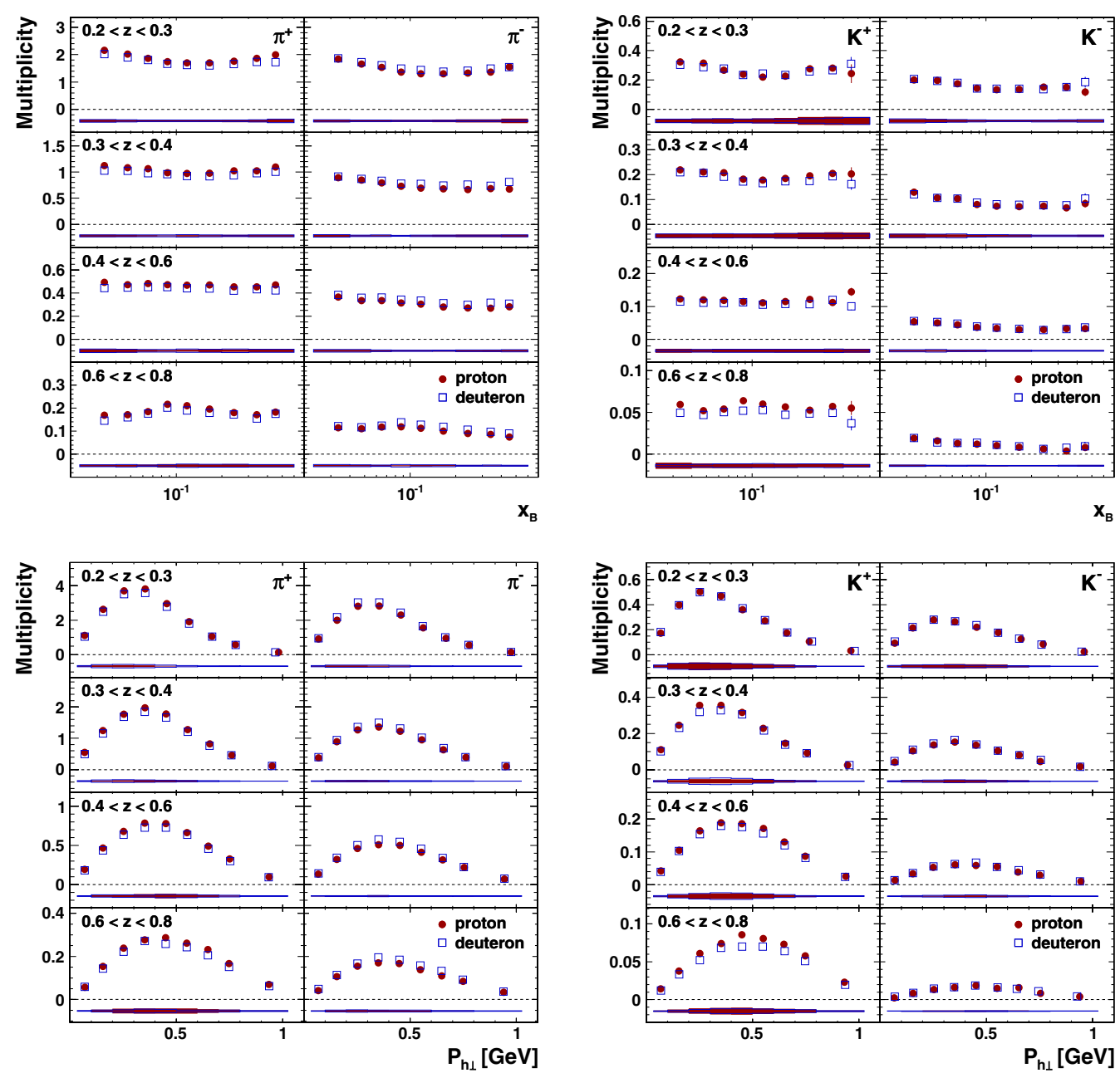

Figure 2. Multiplicities of pions (left panels) and kaons (right panels) for the proton (full circles) and the deuteron (empty squares) as a function of $x_{B}$ and $P_{h \perp}$ in four $z$ bins. Positive charges are on the left and negative charges are on the right of each panel. The systematic uncertainties are given by the error bands.

increasing $z$ in the range $0.2<z<0.8$, reflecting the fact that $K^{-}$cannot be produced through favored fragmentation from the nucleon valence quarks. In Fig. 2 the multiplicities are presented for positively and negatively charged pions and kaons as a function of the Bjorken $x_{B}$ and the transverse momentum of a hadron. The features of the $P_{h \perp}$ distributions result from the combined effects of the initial transverse motion of the struck quark, of the emission of soft gluon radiation during the scattering process, and of a transverse momentum component which is generated during the fragmentation process.

\section{References}

[1] J.C. Collins, L. Frankfurt, and M. Strikman, Phys. Rev. D 56, 2982 (1997)

[2] S. Albino, B.A. Kniehl, G. Kramer, and C. Sandoval, Phys. Rev. D 75, 034018 (2007)

[3] K. Akertaff et al., Nucl. Instrum. Methods A 417, 239 (1998)

[4] N. Akopov et al., Nucl. Instrum. Methods A 479, 511 (2002) 